\title{
PATH DURATION ANALYSIS IN VEHICULAR AD HOC NETWORK
}

\author{
R. S. Raw, Vikas Toor, and N. Singh \\ Ambedkar Institute of Advanced Communication Technologies \& Research \\ Delhi, India \\ rsrao08@yahoo.in, vikastoor@gmail.com, \\ nsingh1973egmail.com
}

\begin{abstract}
In Vehicular Ad hoc Networks (VANETs) the mobility of the nodes is the main concern. This mobility of nodes makes the route unstable and unreliable for the information exchange and communication between two nodes in the network. To enhance the performance and throughput of the VANETs, routes between nodes must be reliable and stable. In this paper, we study the significance of path duration and link duration in Vehicular Ad hoc Networks (VANETs). Because of this mobility, connectivity graphs changes very frequently and it affects the performance of VANETs. Therefore, path duration can be used to predict the behaviour of the mobile nodes in the network. Estimation of the path duration in VANETs can be a key factor to improve the performance of the routing protocol. Estimation of path duration is a challenging task to perform as it depends on many parameters including node density, transmission range, numbers of hops, and velocity of nodes. This paper will provide a comprehensive study for estimating the path duration in VANETs.
\end{abstract}

\section{KEYWORDS}

VANET, MANET, Path Duration, Link Duration, Least Remaining Distance, Routing Protocols.

\section{INTRODUCTION}

VANET is a subclass of Mobile Ad hoc Networks (MANETs). VANETs are special in the sense of their mobile nodes, these nodes are vehicles on the roads and the mobility of these vehicles is very high. VANETs are real time network, used in the daily life or practically on the roads. The increasing traffic on the roads causes accidents and a matter of concern worldwide. Increasing vehicular crime is also an important issue for the nations worldwide. These accidents and crimes cause loss to life, economy, and security of human beings. VANET is one of the best solutions for all these problems. VANETs will help in making the road safer and well organized in future. The Intelligent Transportation System (ITS) has been working since long time to make the road safe, secure, and has improved efficiently in way to travel and transportation [1]. 802.11 WLAN technologies developed a Dedicated Short Range Communication (DSRC) in 2003 under the Federal Communication Commission (FCC) for VANET. DSRC service is using the 5.850-5.925 $\mathrm{GHz}$ for the communication between vehicles-to-vehicles (V2V) and vehicles to road-side units (V2R). 


\subsection{Comparison of MANETs and VANETs}

VANETs are the special subclass of MANETs but some of the parameters are very different in VANET when we do comparison. To study the estimation of path duration in VANETs, these differences in parameters should be clear. Because the estimation of path duration is depends on various designing parameters of the VANETs. Although both MANETs and VANETs are ad hoc networks but the routing protocols of MANETs are not feasible with VANETs. VANETS are fast moving ad hoc network, so MANETs routing protocols are not able to provide the maximum throughput with VANETs. The study of comparison of MANETs and VANETs is as under in the Table 1.

Table 1. Comparison of MANETs and VANETs [2].

\begin{tabular}{|l|l|l|l|}
\hline S. No. & \multicolumn{1}{|c|}{ Parameter } & \multicolumn{1}{|c|}{ MANETs } & \multicolumn{1}{|c|}{ VANETs } \\
\hline 1. & Cost of production & Cheap & Expensive \\
\hline 2. & Change in n/w topology & Slow & Frequent and very fast \\
\hline 3. & Mobility & Low & High \\
\hline 4. & Node Density & Sparse & $\begin{array}{l}\text { Dense and frequently } \\
\text { variable }\end{array}$ \\
\hline 5. & Bandwidth & Hundred Kps & Thousand Kps \\
\hline 6. & Range & Upto 100m & Upto 500m of \\
\hline 7. & Node Lifetime & $\begin{array}{l}\text { Depends on power } \\
\text { resource }\end{array}$ & $\begin{array}{l}\text { Depend on } \\
\text { vehicle }\end{array}$ \\
\hline 8. & Multi-hop Routing & Available & Weakly available \\
\hline 9. & Reliability & Medium & High \\
\hline 10. & Moving pattern of nodes & Random & Regular \\
\hline 11. & Addressing scheme & Attribute Based & Location based \\
\hline 12. & Position Acquisition & Using Ultrasonic & Using GPS,RADAR \\
\hline
\end{tabular}

\subsection{VANETs Applications}

VANETs make road secure by setting a communication between vehicles or their drivers and concerned authorities in periodic manner. VANETs will provide us following applications [3]:

\subsubsection{Infrastructure to vehicle applications}

- Violation warning

- Vehicle location information

- Back up route information

- Road blockade alarm

\subsubsection{Vehicle-to-vehicle applications}

- Electronic brake warning

- Oncoming traffic warning 
- Vehicle stability warning

- Lane change warning

- Collision warning

VANET is a Vehicle-to-Vehicle (V2V) and Vehicle to Road-side units (V2R) communication system [1]. In V2V communication, vehicle exchange the information about each other's status. In V2R communication, road-side units exchange the information with vehicles about the traffic flow and route information. In VANETs, due to lack of any predetermined infrastructure, nodes are acting as a router in the network. These nodes can communicate with each other through multiple paths using direct links within the transmission range or using intermediate nodes to transfer data packet from source to destination. VANETs has all the characteristics of the MANETs but the key difference is that it has come from the high speed and uncertain mobility of the nodes along the network. VANET nodes have battery backup from the vehicles battery power, so there is no power management issue in VANETs. The mobility and density of nodes changes with time and location, like the speed of nodes is higher on highways in comparison to city roads. Density of nodes is higher in daytime in comparison to night; these factors always affect the topology and connectivity graph of the network.

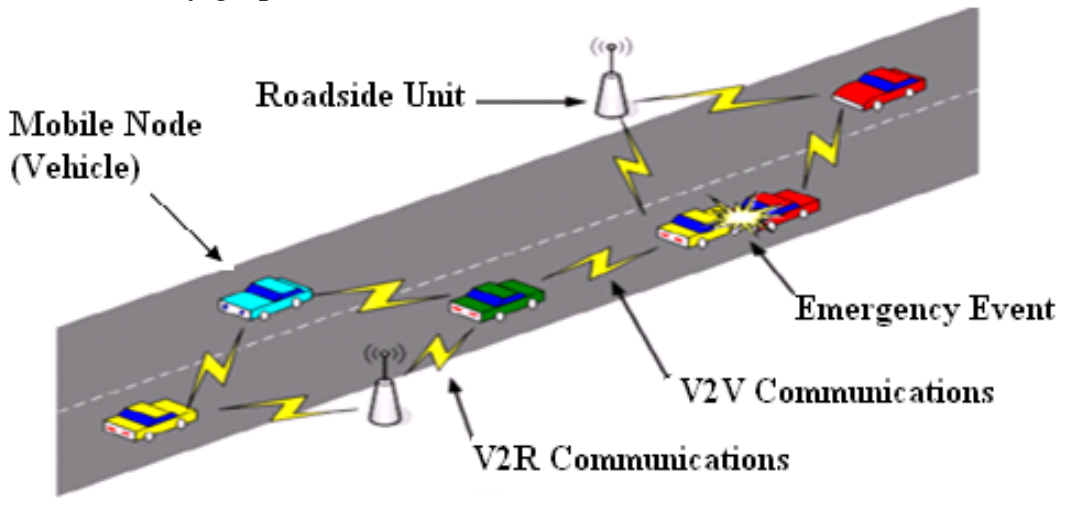

Figure 1. VANETs Communication Scenario [4]

In VANETs, the method of selecting intermediate node, maintenance of path and recovery of path is depends on the characteristics of the routing protocols. The ad hoc networks protocols can be classified in to three categories: table driven, on demand and hybrid protocol [5]. Table driven protocol are proactive and static in nature. In Table driven protocols node exchange periodically messages to update the information of neighbouring nodes and topology of the network. So, if new route is required, it can be immediately provide with the help of information stored or collect via periodic messages. on the other hand, On demand protocols are reactive and dynamic in nature. In on demand protocols, the routes are generated only when connection is require or requested. Finally, hybrid protocol combine the advantages of both table driven and on demand protocols, for a close restricted area the scheme of the table driven protocol is used and for the outer area which is not in the transmission range of the source node, scheme of on demand protocols used [6]. Any one of the above explained protocol used in the VANETs for the routing and communication purpose. The path duration is the amount of time for which the path is active and available or till any of the link goes down. Link duration is the amount of time for which two nodes are connected directly, without any intermediate node. The performance of the VANETs can be enhanced by providing stable and reliable links between nodes. In VANETs, path failure occurs very frequently and main reason is link breakage. In this paper, we analysis the different factors affecting link duration and stability; also compute the reliability of the link in VANETs [5]. 
International Journal on AdHoc Networking Systems (IJANS) Vol. 2, No. 4, October 2012

The rapid change in the topology is a big problem for routing protocol in VANETs. As we know, routing is the process of finding the optimal path between source and destination node and then sending message in a timed manner [7]. As said earlier in this paper the route between source and destination can be of single hop (direct) or multi hops (intermediate) in ad hoc multi-hop network. The knowledge of relative position of nodes is very useful in delivering the message from one node to other nodes. The mobility of nodes and rapidly changing topology of the network creates problem in maintenance of routes in VANETs. Due to mobility and frequent change in network topology, one or more links along a path goes down, the path becomes invalid. This affects the on-going communication and increase the overhead. Frequent path breaks degrade the performance and efficiency of the network [7].

Path duration is the amount of time, the path stays available till one of the link along the path goes down [5]. If we are able to calculate or estimate the path duration of the routes in VANETs, this can help to deal with many challenges of the VANETs. As path duration is an important performance measure parameter, that can improve the performance of the network.

The rest of the paper is organized as follows. Section 2, present the literature survey of the path duration in ad hoc networks. Section 3, present the path duration analysis. Finally, section 4 conclude this paper.

\section{LITERATURE SURVEY}

Significant work has been carried out in the estimation of the path duration in ad hoc networks. Several theoretical and mathematical works related to the estimation of path duration and different parameters which depends on it directly or indirectly has been done.

Estimation of path duration in the MANETs is problematic. In VANETs estimation of path duration becomes more difficult as the speed of the nodes is very high. As we have seen in MANET, intermediate node is critical to send the data from source to destination. The number of hops present between source and destination is the key element to calculate the path duration in the MANET like VANETs. In [8], an analytical approach to calculate the numbers of hops between source and destination and Euclidean distance in the uniformly distributed nodes through greedy forwarding are proposed. Greedy forwarding is also known as least remaining distance (LRD) that attempts to minimize the remaining distance to the destination with each hop. In [8], the average distance and progress per hop gradually varies with respect to current distance to the destination node and is a function of node density. The idea of hop count between two nodes with the Euclidean distance helps in the estimation of end to end delay with the help of per hop transreceive latency.

In [5], in order to maximize the path duration in MANET, a scheme is proposed. It tries to maximize the expected path duration of the routes and also perform the path recovery in case of primary path failure by computing probability of a cached alternative path that may be available. Author shows that when the hop count along the path is large, the distribution of path duration can be calculated by an exponential distribution and parameter of the exponential distribution is given by the sum of the inverse of the expected duration of the links along the path. Secondly, avoiding nodes with shorter average link duration can help to choose the long lasting paths.

In [9], analysis of the path duration statistics and their impact on reactive MANETs protocol has been done for better understanding of the mobility in the network. It has been observed that the path duration, depends on Path Density Functions (PDFs) for the path of two or more hops can be approximated by an exponential distribution, which is parameterized by the relative speed of the mobility model, transmission range of the node, and number of hops in the path. In [10], the behaviour of the communication links of a node in a multi-hop environment has been presented. 
International Journal on AdHoc Networking Systems (IJANS) Vol. 2, No. 4, October 2012

In which, an approach is provided for better understanding the behaviour of communication links in the presence of mobility or mobile nodes. In [11], the performance comparison of different position-based routing protocols in VANETs has been done and it shows that the features and concepts of routing protocol used. This provides us with a great help in choice of the right routing protocol and made calculation for the estimation of the path duration. This comparison also helps to choose the best routing protocol for the calculation of the path duration in VANETs.

In [12], the distribution of path durations in MANETs is studied with the help of Palm's Theorem. Under a set of mild conditions, the distribution of path duration converges to an exponential distribution with appropriate scaling, as the number of hops increases. In [13], authors formulate the problem of optimal next-hop selection in a route between two vehicles on highway. In this, author also try to find the optimal number of hops in one link with the help of the optimal selection of next-hop in the maximum route lifetime based on vehicle speed and internode distance. To get the optimal path and expected lifetime, author propose a solution where two vehicles are moving on the highway. However, this research only focuses on one direction only. They ignore the scenario of opposite direction of vehicle movement.

\section{ANALYSIS OF PATH DURATION IN VANETS}

Path duration is primarily based on the path selection as only after selecting a path, other parameters comes into the scenario of estimation. Therefore, the path selection is also an important aspect in VANETs. Path selection should be done, so that the distance between the source and destination should be minimum. However, in [6], a scheme to explore long lifetime of a link is proposed, which shows that the shortest path is not the best path when path duration is taken into account. The path duration of the route is also critically essential as the path breakage affects the communication in the mid of the transmission. A new route or path has to be set up for the further communication once a path failure happens. It degrades the performance of the ad hoc networks, as new route requires time and overhead both. To increase the efficiency and performance of the VANETs, knowledge of the path duration can help greatly. The path duration is also not an independent factor as it also depends on various other factors [9]. The parameters, which related to the path duration are examined under different models and protocols.

\subsection{Path duration under different ambience}

In this paper, we focus on the study of the path duration in the VANETs. The path duration in MANETs is analyzed under different mobility model and routing protocol. Every mobility model and protocol shows some common parameters, which should be followed to calculate the path duration in ad hoc networks. The path duration is an effective and important factor to design a new routing protocol, which has high efficiency and throughput with less number of link breakage and high transmission rate.

Random waypoint mobility model is a free flow model where nodes are moving in random directions. Distribution of path duration in MANET using Random Waypoint Mobility Model has been carried out in [12]. It shows that under a set of mild conditions, the path duration distribution can be estimated by an exponential distribution as the number of hops along the path increases. Mathematically, it computes the link duration distribution with given speed of node and provides the correlation of the residual life of links. Finally, the result shows that the inverse of path duration can be estimated accurately by the sum of the inverse of the link duration of the links along the path, when number of hops is large.

Greedy Routing [8] approach in ad hoc networks is based on the Least Remaining Distance (LRD) forwarding method. This is one of the most used methods in the MANETs for the 
transmission of data in multi-hop routes. In LRD forwarding method, a forwarding node finds the position information of direct neighbours within the transmission range and selects one of these nodes, which is closest to the destination node as the next-hop node [14]. In others words, LRD selects the next hop node that attempts to minimize the remaining distance to the destination within transmission range of the source node with each hop. Greedy routing tries to cover the maximum distance of the route per hop in the multi-hop transmission and provide estimation of average distance progress per hop. In [8], authors shows that the node density and current distance to the destination is the function of average progress per hop and with the given hop count; the bounds on Euclidean distance can be computed numerically.

Ad hoc On-demand Distance Vector Routing Protocol (AODV) is the reactive routing protocol and selects the first available path. Distribution of path duration in MANETs using AODV has been carried out in [15]. In this schema, each node maintains a sequence number and broadcast ID. Here, sequence number shows the freshness of the path request. To avoid path failure, routes are ranked according to destination sequence number and inverse path duration values. All these above factors are related to link duration and hop counts. The path with the largest expected path duration can be calculated by the exponential distribution, when links are dependent and heterogeneous.

To calculate the path duration in VANETs, Border node based most Forward within Radius (BMFR) is one of the best option. B-MFR [13] avoids using the interior nodes within the transmission range for packet forwarding. In B-MFR forwarding, a packet is sent to the next-hop node, which is present on the border of the transmission range towards the destination. The border node with the greatest progress on the straight line is chosen as next-hop node for transmitting packets further. Therefore, B- MFR forwards the packet to the border node that is closest to the destination node and attempts to minimize the number of hops. In B-MFR, the expected distance, expected number of hops between source and destination, and maximum progress towards destination is also mathematically estimated. These mathematical expressions are very useful and helpful in estimation of path duration in VANETs.

Edge node based Directional Routing Protocol (E-DIR) [16] is position based protocol, which is more suitable protocol for dense networks, where number of nodes per unit area are enough to provide connectivity. The edge node with smallest angle towards the destination is chosen as the next-hop node. The nodes should be present at the border area for the source node to send the data packet from source to destination as E-DIR uses the edge nodes for the next-hop transmission towards the destination. In this protocol, expected number of hops between source and destination are calculated mathematically. In E-DIR, numbers of hops are effectively minimized as it uses the edge nodes. Therefore, E-DIR shows that the density of nodes in the network is also an important factor for designing of new routing protocols so that these can increase and enhance the performance and efficiency of the networks.

In [17], we study the real world, practically collected data gathered from 20 user in real mobile ad hoc network for the analysis of the residual lifetime of the links and paths in the network. The experiment compares the result of the data with the two very commonly used mobility models for MANETs including Random Waypoint and Random Reference Group Mobility Model. The result shows that mobility of nodes and number of hops effects the lifetime of the links and routes. It also calculates the conditional route lifetime CDF (Cumulative Distribution Function) for routes, mathematically.

In [18], author analysed the connectivity in wireless ad hoc networks using graph-theoretic approach. The result shows that graph theoretic approach could be much preferable if there is line of sight communication in the networks. The transmit power or connectivity can be degrades in graph theoretic approach if there is multipath fading or multiple access interference in the 
network. Link connectivity can be gained with graph theoretic approach in the line of sight communication. In [19], the beam forming antenna concept is used to enhance the connectivity limits. The result shows that connectivity of the ad hoc networks could be improved with adaptive antennas, by providing the optimal beam directions. This work is important from the view of connectivity in VANETs.

In [14], the path duration is estimated for the MANETs. It shows that path duration is not easy to estimate as there are many other parameters which are related to it. In [12], mathematical model is proposed and validated with the help of the simulation process. In MANETs, the mobility and speed is comparatively low than the VANETs. However, the mathematical model can help in many ways. It can provide the base and motivation to estimate the path duration in MANETs as well as in VANETs.

\subsection{Parameters for calculations}

As per the analysis, the parameters on which path duration of the multi-hop routes in VANETs depends are as follows:

\subsubsection{Least Remaining Distance and Shortest Path}

In shortest path, we choose the next-hop node, which is at shortest distance to the destination and provide least number of hops. Similarly, in least remaining distance forwarding, choose the nexthop node, which is closest to the destination and has minimum remaining distance to the destination [8].

\subsubsection{Link Residual Life}

Link Residual Life is the time for which the direct link between two nodes is active and is a part of the route. Links are as far as both of nodes (source and next-hop node) in the transmission range of each other [18]. It can be defined as:

$$
t=\frac{d}{v_{r}}
$$

$d$ is the distance between next-hop node and maximum transmission range of source node, $v_{r}$ is the relative velocity of source node and next-hop node.

\subsubsection{Link Distance}

Distance between two nodes, which provide a link to a route can be defined as the link distance. Link distance depends on the protocol which is used in the VANETs. Link distance will increase if we choose the border node of the transmission range as a next-hop node.

\subsubsection{Node Density}

Node density means number of vehicles per unit area of the transmission range. It affects the path duration as if we have sparse number of nodes. In this case, link formation towards the destination is a difficult job. If we have high node density network and we choose the border node as a next-hop node then it will suffer with the Edge Effect [14]. When any border node is moves out from the transmission range of the source node and path failure occurs, then it is known as Edge Effect in the network. 


\subsubsection{Velocity of Nodes}

Direction of motion of a node and its speed both are crucial for the calculation of the path duration between the nodes. Velocity of nodes should not cause the link breakage or path breakage in VANETs as it can take the next-hop node out of transmission range. Link duration is also depends on the relative velocity of the nodes as it can increases the link distance. Equation (1) shows that relative velocity between nodes, which is inversely proportional to the link duration.

\subsubsection{Number of Hops}

Number of hops can be defined as the number of intermediate nodes in the route (source to destination). Number of hops depends on all the above discussed parameters. As every parameter, contributes to decide the number of hops between source and destination. Number hops should be as low as possible which decrease the chances of link breakage.

\subsubsection{Average Progress per Hop}

Average progress per hop is the average distance covered by the each hop present in the route. More the average progress per hops means less the number of hops. If there is less number of hops, it means there is less number of links and less chances of link breakage.

As we have seen, all the parameters are inter-related with each other as well as to the path duration of the multi-hop routes in VANETs. This shows that, in order to calculate the path duration in VANETs, these above parameters are crucial and must be considered in the calculations.

\section{CONCLUSION}

The path duration in VANETs is a key design parameter, which can be useful to improve the performance and throughput of the network. The path duration will be helpful in the process of path selection and for the transmission of packet from source to destination. But the mobility and uncertainty of the nodes in the VANETs is the most challenging issue for the calculation. Mobility changes the network topology and connectivity graph. Routing protocol has to find a route once again as soon as the route becomes a failure due to mobility. Once a route breaks, the communication is failed between source and destination, which affects the performance of the VANETs. Therefore, the estimation of the path duration for a particular route will provide the information and help to choose a suitable path for the transmission.

The path duration of the route can be calculated by considering the design parameters like node density, transmission range, number of hops and velocities of nodes etc. which affect the path duration of the VANETs. In future, path duration can be estimated in VANETs analytically by considering the above-discussed parameters and routing protocols. 


\section{REFERENCES}

[1] K. Lakshmi, K. Thilagan, K. Rama, A. Jeevarathinam, and S. M. Priya,(2012) "Comparison of Three Greedy Routing Algorithm for Efficient Packet Forwarding in VANET", IJCTA, vol. 3(1),146151,Jan-Feb.

[2] T. H. Kim, W. K. Hong, H. Kim,(2007) "An effective multihop broadcast in vehicular ad hoc networks", proceedings 20th International Conference on Architecture of Computing Systems, ARCS 2007, March, springer.

[3] H. Hartenstein and K.P. Laberteaux,(2010) "VANET: Vehicular Applications and Inter-Networking Technologies", Book of A John Wiley and sons ltd publication.

[4] J. Rao, (2008) "Security in Vehicular Ad hoc Networks (VANETs)", CSE 825: Course Presentation, March 10.

[5] Yijie Han and Richard J. La, (2006) "Maximizing Path Durations in Mobile Ad-Hoc Networks. Information science and system, 40th annual conference on March.

[6] Tarik Taleb, Ehssan Sakhaee, Abbas Jamalipour, Kazuo Hashimoto, Nei Kato, and Yoshiaki Nemoto, (2007) "A Stable Routing Protocol to Support ITS Services in VANET Networks", IEEE Transactions on Vehicular Technology, Vol. 56, NO. 6, November.

[7] Z. Cheng and W.B. Heinzelman, (2004) "Exploring long lifetime routing in ad hoc networks", 7th ACM international symposium on modeling, analysis and simulation of wireless and mobile systems.

[8] S. De. A. Caruso, T. Chaira, and S. Chessa,(2006) "Bounds on hop distance in greedy routing approach in wireless ad hoc networks" Int. J. Wireless and mobile computing, vol. 1, no. 2, pp. 131140, Feb.

[9] N. Sadagopan, F. Bai, B. Krishnamachari and A. Helmy,(2003) "PATHS: analysis of PATH duration Statistics and their impact on reactive MANET routing protocols", proc. Mobihoc, pp-245-256.

[10] P. Samar and S. B. Wicker,(2004) "On the Behavior of Communication Links of a Node in a MultiHop Mobile Environment”, MobiHoc, PP. 145-156, May 2004.

[11] R. S. Raw, S. Das, (2011) "Performance comparison of position based routing protocols in vehicle-tovehicle communication", International Journal of Engineering Science and Technology (IJEST), Vol. 3 No. 1, pp 435-444.

[12] Y. Han, R. J. La, and A. M. Makowski, (2004) "Distribution of path durations in mobile ad hoc networks - Palm's Theorem at work", ITC Specialist Seminar on performance Evaluation of Wireless and Mobile Systems (ITCSS).

[13] R. S. Raw, and D. K. Lobiyal, (2010) “B-MFR Routing Protocol Vehicular ad hoc networks", IEEE, ICNIT, Philippines, Manila.

[14] M. Srinivasan, (2008) "Analytical estimation of path duration in mobile ad hoc networks", Department of Electrical and computer Engineering, Wichita State University, May.

[15] Richard J. La and Yijie Han, (2007) "Distribution of Path Durations in Mobile Ad-Hoc Networks and Path Selection", IEEE/ACM Transactions on networking, vol. 15, no. 5.

[16] R. S. Raw, and D. K. Lobiyal, "E-DIR: a directional routing protocol for VANETs in a city traffic environment", Int. J. Information and Communication Technology (IJCT), ISSN: 1466-6642, Vol. 3 Issue 2, pp. 242-257.

[17] V. Lenders, J. Wagner, and M. May, (2006) "Analyzing the Impact of Mobility in Ad Hoc Networks" ACM, Conference in Florence, Italy.

[18] N. N. S. Kamaruzaman and H. Hasbullah,(2008) "Silent Alarm: Path Optimization Route Lifetime for VANET Multi-Hop Routing Protocol” International conference on network applications, protocols and Services, (Netapps 2008).

[19] S. Panichpapiboon, G. Ferrari and O. Z. Tonguz, (2010) "Connectivity of ad hoc wireless networks : an alternative to graph-theoretic approaches", wireless network, volume 16, Issue 3, April. 


\section{Authors}

Dr. R. S. Raw received his B. E. (Computer Science and Engineering) from G. B. Pant Engineering College, Pauri-Garhwal, UK, India and M. Tech (Information Technology) from Sam Higginbottom Institute of Agriculture, Technology and Sciences, Allahabad (UP), India in 2000 and 2005, respectively. He has completed his Ph. D (Computer Science and Technology) from School of Computer and Systems Sciences, Jawaharlal Nehru University, New Delhi, India in 2011. He is currently working as Assistant Professor at Computer Science and Engineering Department, Ambedkar Institute of Advanced Communication Technologies \& Research, Delhi, India. His current research

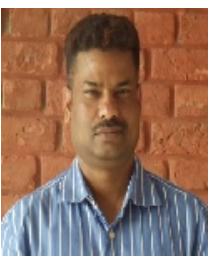
interest includes Mobile Ad hoc Networks and Vehicular Ad hoc Networks. Dr. Raw has published papers in International Journals and Conferences including IEEE, Springer, Inderscience, IERI Communications Letters, American Institute of Physics, AIRCC etc..

Mr. Vikas Toor received his B. Tech. (Information Technology) from Guru Premsukh Memorial College of Engineering, Delhi, India in 2005 and he is pursuing M. Tech (Information Security) from Ambedkar Institute of Advanced Communication Technologies \& Research, Delhi, India. His area of interest includes Mobile Ad hoc Networks and Vehicular Ad hoc Networks.

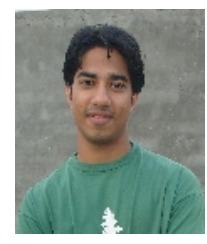

Dr. Nanhay Singh, working as Associate Professor in Ambedkar Institute of Advanced Communication Technologies \& Research, Govt. of NCT, Delhi-110031 (Affiliated to Guru Gobind Singh Indraprastha University, Delhi) in the Department of Computer Science \& Engineering. He received his Ph.D (Computer Science and Technology) \& M. Tech. (Computer Science \& Engineering) from the Kurukshetra, University, Kurukshetra, Haryana. He has rich experience in teaching the classes of Graduate and Post- Graduate in India. He has contributed to numerous International journal \& conference publications in various areas of Computer Science. He published more than

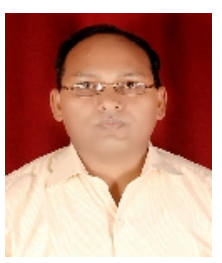
11 Research Paper in International Journals and Conferences. . He has also written an International book Titled as "Electrical Load Forecasting Using Artificial Neural Networks and Genetic Algorithm", in Global Research Publications New Delhi (India). His area of interest includes Distributed System, Parallel Computing, Information Theory \& Coding, Cyber Law, and Computer Organization. 